\title{
Rib kinematics analysis in oblique and lateral impact tests
}

\author{
DOMINIK JASTRZĘBSKI*, RAFAŁ PERZ \\ Institute of Aeronautics and Applied Mechanics, Warsaw University of Technology, Warsaw, Poland.
}

\begin{abstract}
Purpose: Understanding thorax kinematics and rib breaking mechanisms in conditions of oblique and lateral impact is crucial in safety systems development. To increase knowledge level on this subject, simulation and experimental tests are necessary. The purpose of this study was to obtain single rib kinematics in the case of oblique and lateral impact conditions using numerical simulation approach. Methods: Two impact tests using human body model of a 50th percentile man (THUMS v4.0.1 AM50) were performed in LS-Dyna R7.1.1. Impactor was a rigid cylinder with a diameter of $152 \mathrm{~mm}$, and velocity equal to $6.7 \mathrm{~m} / \mathrm{s}$. Impact angle measured to sagittal plane was 30 and $90^{\circ}$, respectively in oblique and lateral impact case. Results: Kinematics of ribs from 3rd to 6th were analyzed. Results shown significant similarities between oblique impact and kinematics of ribs tested in frontal impact conditions in the literature, with maximal costochondral joint displacement relatively to costovertebral joint varying from $65.4 \mathrm{~mm}$ (3rd rib) to $82.0 \mathrm{~mm}$ (5th rib). Deformation of rib in lateral impact conditions was different than during oblique impact test, with distinctive "flattening" approximately in the middle of the rib. Maximal relative displacement varies from $16.4 \mathrm{~mm}$ (6th rib) to $26.6 \mathrm{~mm}$ (5th rib) and its location depends on the analyzed rib. Conclusions: Oblique impact scenario may be simulated for the single rib on an experimental way using set-up of the frontal impact. Experimental simulation of the lateral impact for the single rib should not use the same set-up, as the kinematics analysis showed significant differences between simulated cases.
\end{abstract}

Key words: chest, impact, fracture, thorax, rib, THUMS

\section{Introduction}

Despite the development in road safety, the use of active safety systems and awareness-raising campaigns, car accidents are the 8th leading cause of death on the world (for people of all ages) and the 1st leading cause of death among children and young people (5-29 years old). Number of fatalities reached 1.35 million in 2016 [17].

According to the statistics for Polish roads, lateral and frontal crashes were the cause of $37 \%$ of fatalities and 45\% of injured in all crashes in 2018 [21]. Most European statistics put thorax in the first place of the most common injured body regions (with frequency of 26-39\% of MAIS3+ injuries) [3]. In 2008, Kent et al. [8] has proved, that presence of rib fractures is associated with a significant increase of fatality risk.
To minimize injuries occurring to the thorax, there is a necessity of understanding mechanisms of injuries formation. This understanding may be achieved by performing simulations using different Human Body Models (HBM). However, even though there are theories and numerical models designed for the rib fracture prediction, they still need improvement, as they predictions have errors exceeding 30\% [3]. Another approach to understand the rib fracture mechanisms is an experimental way. Complex body experiments are demanding in interpretation and despite their advantages, number of variables may be too high to find variables with a high influence on the result of the experiment. To prevent that, experiments on body parts can be performed. In the case of ribs, three types of experimental set-ups can be found in the literature: three-point bending, tensile loading [1], [4] and antero-posterior bending [11], [12]. The first two provide material properties data, but major assumptions

\footnotetext{
* Corresponding author: Dominik Jastrzębski, Institute of Aeronautics and Applied Mechanics, Warsaw University of Technology, ul. Nowowiejska 24, 00-665 Warsaw, Poland. Phone: +48 22234 5397, e-mail: djastrzebski@meil.pw.edu.pl

Received: August 4th, 2019

Accepted for publication: February 10th, 2020
} 
of this tests are: negligible beam curvature and small deformations [11]. Antero-posterior bending can be performed without this assumptions, which allows to take large deformation and nonlinear geometry of the rib into account. This test can be conducted to simulate ribs behavior during frontal crash [12]. As the setup is less complicated than during full body crash test, this test may be useful in understanding mechanisms of rib breaking. In the literature there is no kinematics analysis of ribs during oblique and lateral crash, so decision was made to focus on this subject in the following paper.

This paper focuses on a simplified lateral and oblique impact loading conditions to the human thorax, using HBM. The objective of this study was to expand knowledge of the human ribs behavior in oblique and lateral impact conditions. The objective, in particular, was to gain characteristics of ribs movement in oblique and lateral impact cases. A movement characteristic was the position and velocity of the rib specific points in relation to the costovertebral joint, while subjected to impact. These characteristics will be used to develop an experimental set-up of a single rib bending in conditions of tested scenarios. In the future, outcomes of this tests may allow to develop Autonomous Test Devices (ATD) and HBM.

\section{Materials and methods}

For simulations, the Total Human Model for Safety (THUMS) v4.0.1 AM50 was used. It is a finite element model of a whole body, with dimensions close to a 50th percentile man (height: $1.786 \mathrm{~m}$, mass: $74.3 \mathrm{~kg}$ ). It contains of approximately 1.8 million elements. Mostly hexahedron elements were used, with following guidelines: element length $3-5 \mathrm{~mm}$, warpage -50 degrees or less, aspect ratio -5 or less, skew -60 degrees or less, Jacobian -0.3 or more [20]. Pedestrian model was used, due to the license availability, but according to the THUMS documentation, thorax area, which tests are described in this paper is identical in both - pedestrian and occupant version [20]. The thorax of the model was validated for anterior and lateral cylindrical impact and belt loading [20]. There are 1254 materials used in THUMS. As the paper focuses on the ribcage, only materials of ribs and costal cartilage will be described. More detailed materials description can be found in [20] or in the literature.

Rib consists of 2 parts. Outer layer is made of cortical bone, and inner one is a sponge bone. In THUMS, for cortical bone elastic-plastic material was used, with Young Modulus of $13.02 \mathrm{GPa}$, Poisson's ratio 0.3 and Yield Stress $80 \mathrm{MPa}$. Sponge bone is the same material type, with Young Modulus od $40 \mathrm{MPa}$, Poisson's ratio 0.45 and Yield Stress 1.8 MPa. Elasticplastic material was also used for costal cartilage with Young Modulus $29 \mathrm{MPa}$, Poisson's ratio 0.4 and Yield Stress $2.75 \mathrm{MPa}$.

To avoid the discontinuity of rib kinematics due to the costal cartilage damage (which occurred in the 7th costochondral joint), the yield stress of the costal cartilage material was increased.

Two tests schedules were assumed - near side (which means, that the impact approaches from the driver's side) lateral impact and near side oblique impact, both using cylindrical blunt impactor with mass and diameter, made of ridged material similar to Kroell tests [8], [9]. According to the literature, mass of the impactor differs from $1.6 \mathrm{~kg}$ to $23.6 \mathrm{~kg}$ [8] and its velocity from $2.5 \mathrm{~m} / \mathrm{s}$ [15] to $14.5 \mathrm{~m} / \mathrm{s}$ [8]. However, the most common velocity, mass and diameter values found in the literature ([10], [15], [16]) were respectively $4.3-6.7 \mathrm{~m} / \mathrm{s}, 23.4 \mathrm{~kg}$ with diameter of $152 \mathrm{~mm}$.

Setup used for simulations was similar to the setup used by Leport et al. [10] - both oblique and lateral impacts were run with the impactor velocity of $6.7 \mathrm{~m} / \mathrm{s}$ (higher from two used in [10]). Angle between the impact direction and the sagittal plane was $30^{\circ}$ to hit the costochondral joint. Angle of $30^{\circ}$ (instead of $60^{\circ}$ used in the literature) was used for stronger division between oblique and lateral impact. The impactor axis was aiming between 4 th and 5 th rib during the lateral and oblique impact. As mentioned before - the thorax of the THUMS is identical in a pedestrian and occupant version, so, although in experiments described the literature were performed in the sitting position, the pedestrian model could be used in a standing position. It was unattached to the environment - in that way all forces appeared in a model were a result to inertial forces.

As an output, the kinematics of the characteristic points on 3-6 ribs was collected. The points were a nodes of ribs mesh, chosen in the following way:

- A node closest to the costochondral joint (Fig. 1, point $A$ ),

- A node closest to the costovertebral joint (Fig. 1, point $F$ ),

- A node closest to the line, lying at angle of $30^{\circ}$ to the $A-F$ line, and passing through a point in the middle of the section $A F$ (Fig. 1, point $B$ ),

- A node closest to the line, lying at angle of $150^{\circ}$ to the $A-F$ line, and passing through a point in the middle of the section $A F$ (Fig. 1, point $E$ ), 
- A node at the longest distance from the section $A F$ (Fig. 1, point $D$ ),

- A node lying on the outside surface of the rib, closest to its center of mass (Fig. 1, point $C$ ).

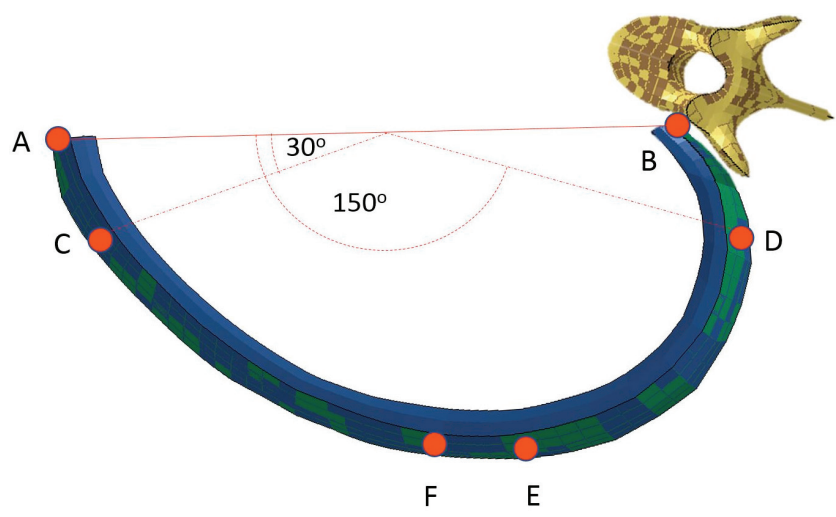

Fig. 1. Top view of the rib with marked characteristic nodes

The displacement of a point A relative to the point $B$ was calculated. Experimental tests, based on the rib kinematics with the use of $A B$ displacement are planned, similar to [12]. Based on this relative displacement, velocities and accelerations were calculated (by numerical integration). For points $C-F$ only relative displacement was calculated, to asses rib shape changes during the impact.

\section{Results}

\subsection{Results of the oblique impact simulation}

In this case, the point of the highest value of relative displacement was a point $A$ lying closest to costochondral joint. Trace of this point may by approximated by combination of 2 functions close to linear, different for ribcage compression and extension (Fig. 2).

\section{Displacement of characteristic nodes of $4^{\text {th }}$ rib, oblique impact}

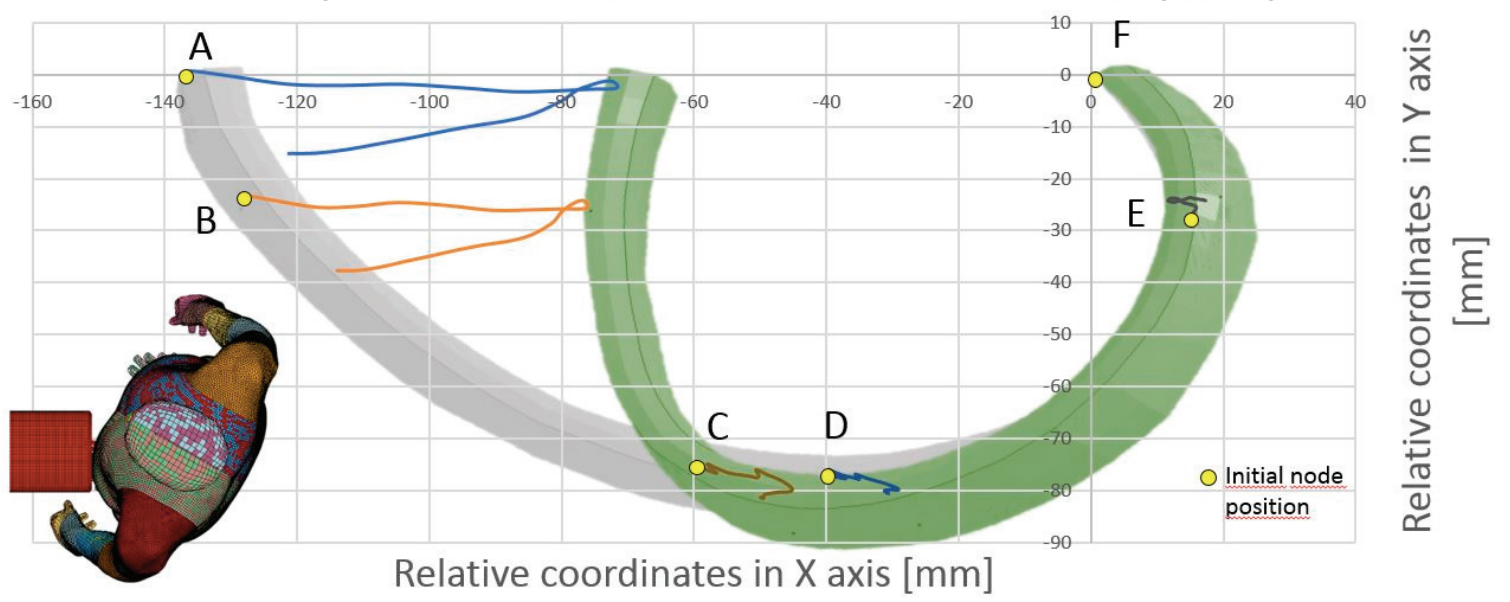

Fig. 2. Displacement of characteristic nodes of 4 th rib during oblique impact simulation

Fig. 3. Displacement of the characteristic nodes in relation to costovertebral joint during oblique impact simulation
Relative displacement of ribs char, nodes (oblique impact)

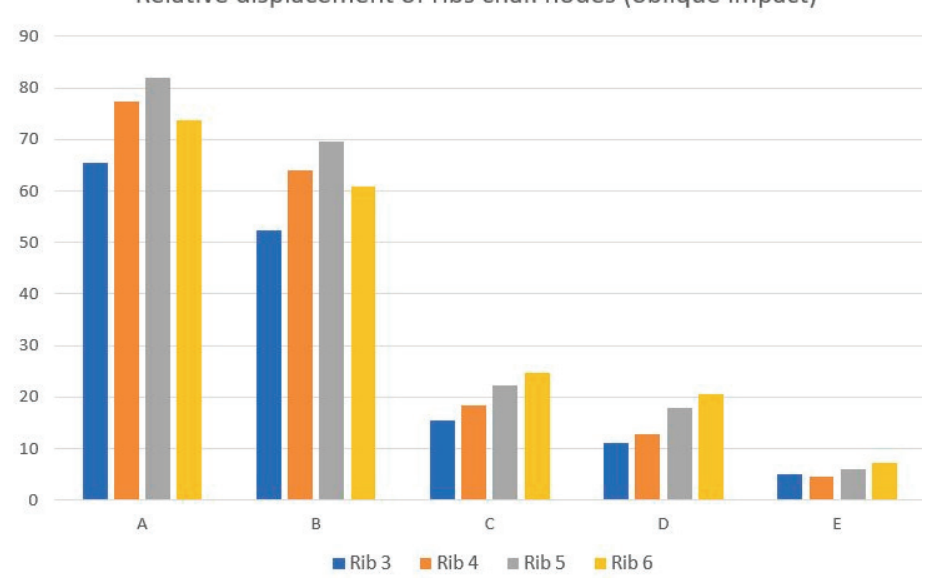


Relative displacements of this point versus the point $F$, for ribs 3-6 were presented in Fig. 3. The character of displacement plots may be find in analysis of simplified simulation of frontal impact [9].

\subsection{Results of the lateral impact simulation}

Neither the nodal traces, nor plots of the maximal deflection of lateral impact simulation may be described in a similar way as during oblique impact scenario. Nodal traces look similar to the loops, which starts with a short linear function for points $A$ and $B$ (Fig. 4). Moreover, for each of the ribs, different characteristic point has maximal displacement. For 3rd rib, it was point A with displacement of $23.9 \mathrm{~mm}$, for rib 4 th - point $C$ with displacement of $25.6 \mathrm{~mm}$, for 5 th rib - point $B$ with displacement equal to $26.6 \mathrm{~mm}$, and for 6th rib points $A$ and $C$ with nearly the same displacement of $16.7 \mathrm{~mm}$ and $16.4 \mathrm{~mm}$, respectively (Fig. 5).

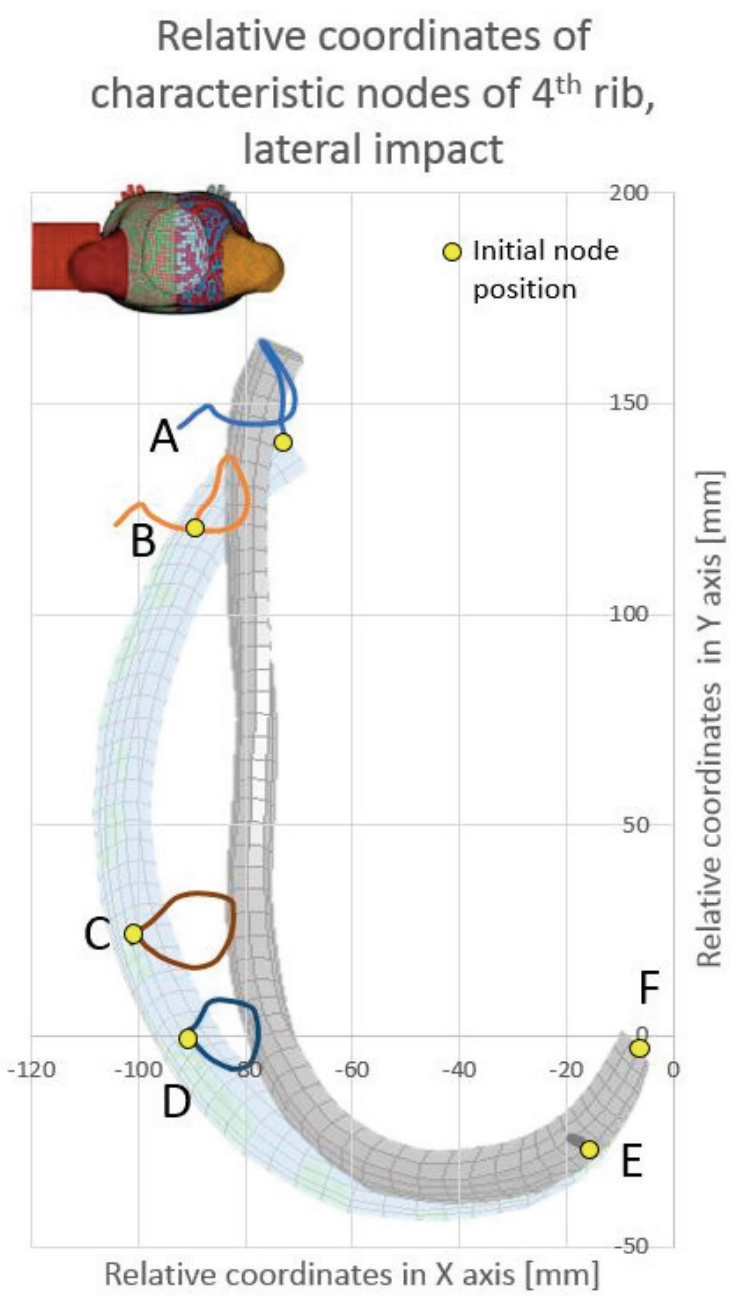

Fig. 4. Displacement of characteristic nodes of 4th rib during lateral impact simulation
Analysis of the whole rib shape during lateral impact simulation brings a conclusion of a distinctive "flattening", which can be find approximately in the middle of the rib (Fig. 4).

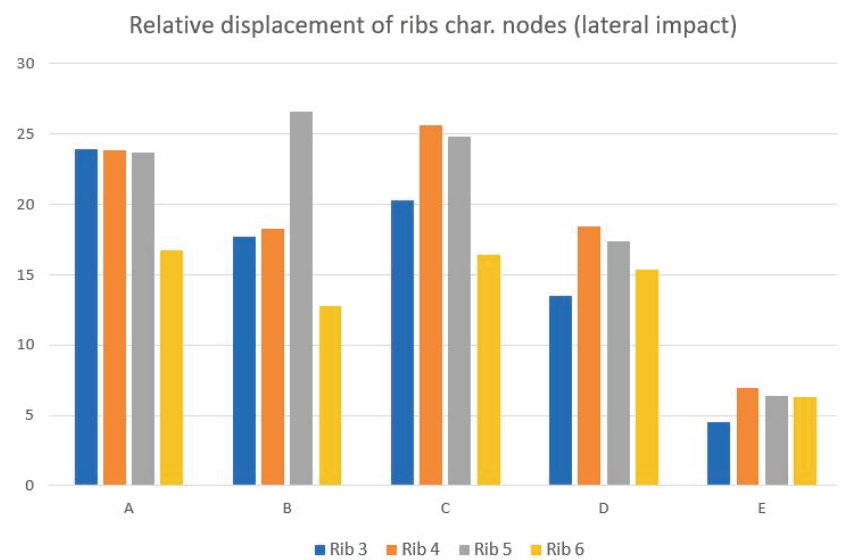

Fig. 5. Displacement of the characteristic nodes in relation to costovertebral joint during lateral impact simulation

\subsection{Result of comparison between oblique and lateral impact case}

Displacement values of the 5th rib were the highest among described ribs. For this reason, 5th rib was chosen to present a comparison between characteristic points displacement during oblique and lateral impact cases. A displacement of characteristic points of 5th rib in two cases was presented in Fig. 6. It is clearly visible that during the oblique impact case displacements of points $A$ and $B$ - two lying close to the costal cartilage - had much higher values. Also, it may be noticed that these two cases have different characters

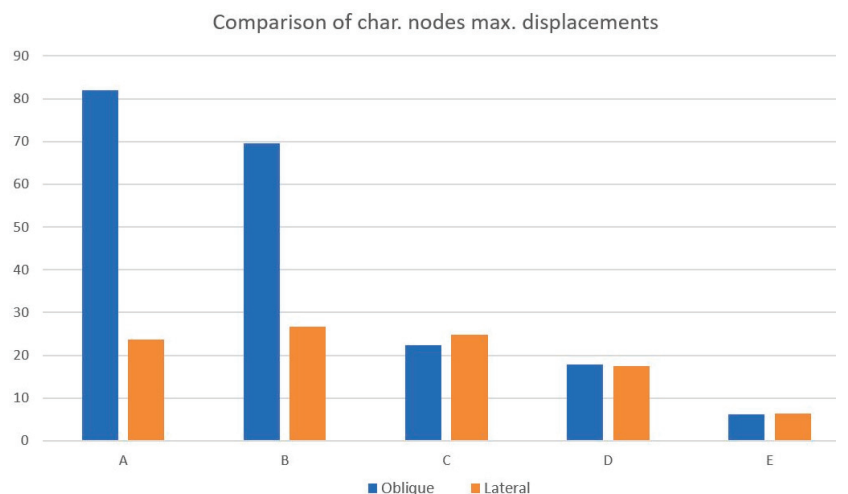

Fig. 6. Comparison of maximal displacements of the characteristic nodes between oblique and lateral impact simulation 
of rib bending. In oblique impact case, there is a dependence between a point maximum displacement value and its placement along rib (the closer to the costal cartilage, the higher is a displacement value). This dependence cannot be find while analyzing the lateral impact case. In this case, maximal displacement of points $A, B$ and $C$ is very similar - 23.7, 26.6, $24.9 \mathrm{~mm}$, respectively, but as it cannot be applied to all tested ribs (Fig. 5), this description is not a dependence.

\section{Discussion}

In relation of increasing amount of oblique and lateral car crashes, research on this subject is important in development of car safety systems. Conducted research has shown that rib behavior during lateral impact differs from the behavior of the rib during oblique impact. However, it occurred that the characteristic of nodal displacement during oblique impact scenario is similar to the same characteristic during frontal impact scenario [12]. Taking into account this similarities and fact that there are set-ups for testing single rib in conditions of frontal crash, it was decided not to focus on the oblique impact scenario.

Objective of the study was to gain characteristics of ribs movement in lateral impact case. The characteristics may be understood as a dependence between node deflection and its position along the rib. This dependence may be very useful to develop an experimental set-up, for single rib testing in described conditions. Using this set-up, ribs may be tested in a simplified environment, which may lead to a deeper understanding of rib breaking mechanism. With this understanding, there is a possibility of using this useful knowledge to improve car safety and decrease level of injuries in the most popular car crash.

Kinematic analysis of the lateral impact simulation has shown no dependence between node deflection and its position along the rib. Therefore, there is a necessity of further, either dynamic or kinematic analysis to achieve described objective. Obtained nodal kinematics combined with the whole rib deformation leads to a conclusion that rib "flattens" during impact. Hence, similarly to the research of Sunnevång et al. [18], it shows that thorax injury criterion based on a chest deflection may be insufficient. Moreover, characteristic "loops" were observed in traces of the chosen nodes. These traces have more sophisticated shape than traces observed in results of the oblique simulation.
Combining flattening of the rib with the nodal kinematics enables to suggest, that set-up used in experiments of a single rib bending [12] would not be compatible with an experimental tests of a single rib bending in a lateral impact case.

In this case, instead of 2 rotational supports (supports which allow rotation of ribs ends around the axis perpendicular to the plane of the rib), a rotational support (near the costovertebral joint) and a deformable one (for the costochondral joint) should be used, while using 2 rotational supports, deflection is implemented by a movement of one of the supports towards the other. It simulates chest deflection in frontal impact case. To simulate chest deformation in the lateral impact case a flexible impactor should be used. Costochondral joint movement may be simulated by using its support made of material with stiffness characteristic comparable with stiffness characteristic of a human ribcage.

In order to estimate the material properties of this support, dynamic analysis of the costochondral joint movement during the lateral impact simulation was conducted. Combining kinematics of point $A$ with Newton equations (1) enabled us to determine mean ribcage stiffness which influence single rib. Mean damping coefficient of the part of the ribcage and its mass which affects rib kinematics was also determined.

$$
\begin{gathered}
m \frac{d^{2} x(t)}{d t^{2}}+b \frac{d x(t)}{d t}+k x(t)=0, \\
\beta=\frac{b}{2 m},
\end{gathered}
$$

where:

$$
\begin{aligned}
& m \text { - mass }[\mathrm{kg}], \\
& k \text { - stiffness }[\mathrm{N} / \mathrm{m}], \\
& \beta \text {-damping coefficient }[\mathrm{Ns} / \mathrm{m}] .
\end{aligned}
$$

Due to nonlinear characteristic of point $A$ relative to $B$ trace, damping coefficient and mass were average of values determined for each timestep.

Results of this analysis lead to a nonphysical values of the damping coefficient. So, although mass and stiffness were a positive, real values, they should not be treated as approvable data.

This analysis showed that previously proposed set-up with one rotational support and one deformable would result in a nonphysical data. Real rib is supported not only on each of its ends, but also along its length on top and bottom side with intercostal muscles, which connects rib with a previous and a following rib (for ribs from 2 to 11). Additionally, each rib is supported by lungs and internal organs on 
the internal side of it, but as organs density and stiffness are much lower than this parameters of muscles, in a simplified test, this support may be omitted.

\section{Conclusions}

Two impact scenarios were analyzed during this research - oblique and lateral impact to the full HBM. Oblique impact simulation results showed many similarities to the frontal impact scenario. Results of the lateral impact simulation varied from the first one in many aspects - from the maximal deflection values, through the characteristic of the rib kinematics to shape of the rib during its maximal deformation.

It was concluded that the oblique impact scenario may be simulated for the single rib on an experimental way using set-up of the frontal impact. This set-up contains two rotational supports on each of the rib ends (with rotation axis perpendicular to the plane containing the rib). Disturbance is implemented with displacement of one of the supports.

Experimental simulation of the lateral impact for the single rib should not use the same set-up, as the kinematics analysis showed significant differences between simulated cases. The experimental set-up should contain of one rotational support, one support made of flexible material (probably stiff rubber) and two long supports, which should be fixed along the rib on the top, and bottom surface (simulating intercostal muscles). The disturbance would be implemented by an impactor aiming approximately to the furthest point from the section joining costochondral and costovertebral joints (previously described as $A F$ section). The impact direction should be perpendicular to this section. Impactor surface should be covered with flexible material to simulate interaction between rib and flesh.

After preparation of a lateral impact experiment, its variations should be taken into account. It is known that even slight change in a position of a head effect on chest shape, and respiratory movements [19], which could suggest that other thorax properties may vary with initial position. Another parameters that should be taken into account are distribution of rib material parameters [14], variation of cortical bone thickness [13], three axial differences in ribs geometry among people [4] and distribution of soft tissues parameters in society [7]. Further analysis and experiments will be a subject of the future research.

\section{References}

[1] Cormier J.M., Stitzel J.D., Duma S.M., Matsuoka F., Regional Variation in the Structural Response and Geometrical Properties of Human Ribs, 49th Annual Proceedings Association for the Advancement of Automotive Medicine, 2005.

[2] European Commission, Study on Serious Road Traffic Injuries in the EU, 2016, https://doi.org/10.2832/29647

[3] Guleyupoglu B., Koya B., Barnard R., GayziK F.S., Failed rib region prediction in a human body model during crash events with precrash braking, Traffic Injury Prevention, 2018, 19, 37-43, https://doi.org/10.1080/ 15389588.2017.1395873

[4] Jastrzębski D., Poulard D., Panzer M.B., Development of Morphed Ribcage Finite Element Models for Comparison with PMHS Data, IRCOBI Conference 2017, 2017, 745-747.

[5] Kemper A.R., Mcnally C., Pullins C.A., Freeman L.J., Duma S.M., Rounana S.W., The Biomechanics of Human Ribs: Material and Structural Properties from Dynamic Tension and Bending Tests, Stapp Car Crash Journal, 2007, 51 (October), 235-273.

[6] Kent R., Woods W., Bostrom O., Fatality risk and the presence of rib fractures, Annals of Advances in Automotive Medicine Association for the Advancement of Automotive Medicine Annual Scientific Conference, 2008, 52, 73-82.

[7] Kozuń M., Kobielarz M., ChwiŁkowska A., Pezowicz C., The impact of development of atherosclerosis on delamination resistance of the thoracic aortic wall, Journal of the Mechanical Behavior of Biomedical Materials, 2018, 79, 292-300, https://doi.org/10.1016/j.jmbbm.2018.01.009

[8] Kroell C.K., SchneIDER D.C., NAHUM A.M., Impact Tolerance and Response of the Human Thorax, Society of Automotive Engineers, Inc., New York 1972, 84-134.

[9] Kroell C.K., SchneIDER D.C., NAHUM A.M., Impact Tolerance and Response of the Human Thorax II, Society of Automotive Engineers, Inc., Warrendale, PA, 1974, 383-457.

[10] Leport T., BAudrit P., Potier P., Trosseille X., Lecuyer E., Vallancien G., Study of Rib Fracture Mechanisms Based on the Rib Strain Profiles in Side and Forward Oblique Impact, Stapp Car Crash Journal, 2011, 55, 199-250.

[11] Li Z., Kindig M.W., Kerrigan J.R., Untaroiu C.D., Subit D., CRAndall J.R., Kent R.W., Rib fractures under anteriorposterior dynamic loads: Experimental and finite-element study, Journal of Biomechanics, 2010, 43(2), 228-234, https://doi.org/10.1016/j.jbiomech.2009.08.040

[12] Perz R., Toczyski J., Kindig M., Ito D., Ejima S., KAMIJ K. et al., Evaluation of the Geometrical Properties Distribution Along the Human Ribs Using Different X-Ray Imaging Methods, IRCOBI Conference 2013, 2013, 245 $-256$.

[13] Perz R., Toczyski J., Subit D., Variation in the human ribs geometrical properties and mechanical response based on $X$-ray computed tomography images resolution, Journal of the Mechanical Behavior of Biomedical Materials, 2015, 41, 292-301, https://doi.org/10.1016/j.jmbbm.2014.07.036

[14] Pezowicz C., Glowacki M., The mechanical properties of human ribs in young adult, Acta Bioeng. Biomech., 2012, 14 (2), 53-60, https://doi.org/10.5277/abb120207

[15] Poulard D., Kent R.W., Kindig M., Li Z., Subit D., Thoracic response targets for a computational model: A hierarchical approach to assess the biofidelity of a 50th-percentile 
occupant male finite element model, Journal of the Mechanical Behavior of Biomedical Materials, 2015, 45, 45-64, https://doi.org/10.1016/j.jmbbm.2015.01.017

[16] Schoell S.L., Weaver A.A., Vavalle N.A., Stitzel J.D., Age- and Sex-Specific Thorax Finite Element Model Development and Simulation, Traffic Injury Prevention, 2015, 16, 57-65, https://doi.org/10.1080/15389588.2015.1005208

[17] Sijabat T.W.S., Global Status Report On Road Safety 2018, WHO, 2018.

[18] SunnevÅng C., Lecuyer E., Hynd D., Carroll J., Kruse D., BoströM O., Evaluation of Near-Side Oblique Frontal Impacts Using THOR with SD3 Shoulder, Traffic Injury
Prevention, 2014, 15, 96-102. https://doi.org/10.1080/ 15389588.2014 .934367

[19] Szczygiel E., Węglarz K., Piotrowski K., Mazur T., Miętel S., Golec J., Biomechanical influences on head posture and the respiratory movements of the chest, Acta Bioeng. Biomech., 2015, 17(2), 143-148, https://doi.org/10.5277/ abb-00118-2014-02

[20] Toyota Motor Corporation, Documentation Total Human Model for Safety (THUMS) AM50 Pedestrian / Occupant Model, 2011.

[21] Wypadki drogowe w Polsce $w 2018$ roku (Car accidents in Poland in 2018, org. in Polish), Komenda Główna Policji, Biuro Ruchu Drogowego, Warszawa 2019. 